\title{
Aumento na detecção de casos de hanseníase em São Luís, Maranhão, Brasil, de 1993 a 1998. A endemia está em expansão?
}

\author{
Increase in leprosy detection rates in São Luís, \\ Maranhão, Brazil, from 1993 to 1998. \\ Is the endemic expanding?
}

Ivan Abreu Figueiredo 1

Antônio Augusto Moura da Silva 2

\footnotetext{
1 Mestrado em Saúde e Ambiente, Universidade Federal do Maranhão. Praça Madre Deus 2, São Luís, MA 65025-560, Brasil. 2 Departamento de Saúde Pública, Universidade Federal do Maranhão. Rua Barão de Itapary 155, São Luís, MA 65020-070, Brasil. aasilva@elo.com.br
}

\begin{abstract}
A descriptive epidemiologic study on the detection of new leprosy cases was conducted in São Luís, Maranhão, Brazil, from 1993 to 1998. A database was created for the purpose, covering 2,796 reported cases. General detection rates were calculated, as well as specific rates by gender, clinical type, and age group. Linear, exponential, geometric, and log adjustment models were performed to analyze time trends in the disease. An increase in detection was observed, involving mostly female and paucibacillary cases, mainly of tuberculoid leprosy. The increase in detection was most evident in the 15 to 19 year-old population. The percentage of detection under 15 indicated the need for active case search in this group.

Key words Leprosy; Descriptive Epidemiology; Communicable Diseases

Resumo Foi realizado estudo epidemiológico descritivo sobre detecção de casos novos de hanseníase em São Luís, Maranhão, Brasil, de 1993 a 1998, a partir da criação de banco de dados com 2.796 notificações. Foram calculados coeficientes de detecção brutos e específicos por sexo, forma clínica e faixa etária. Foi analisada sua tendência temporal por meio dos modelos de ajuste linear, exponencial, geométrico e logarítmico. Evidenciou-se tendência de aumento às custas do sexo feminino e das formas paucibacilares, principalmente da tuberculóide, e mais acentuada na população de 15 a 19 anos. O percentual da detecção em menores de 15 anos indicou necessidade de busca ativa da doença nesta população.

Palavras-chave Hanseníase; Epidemiologia Descritiva; Doenças Transmissíveis
\end{abstract}




\section{Introdução}

Em 1992, o Ministério da Saúde (MS) foi signatário da meta de eliminação da hanseníase proposta pela Organização Mundial da Saúde (OMS). Em 1995, foi formulado o Plano de Eliminação Nacional (PEL) visando a reduzir a taxa de prevalência a menos de um caso para 10 mil habitantes até o ano 2000, em todo o território nacional (MS, 1999).

Os dados mais recentes do MS relativos à prevalência e detecção da hanseníase no Brasil são de 1998. Estes coeficientes têm parâmetros de avaliação determinados pelo Centro Nacional de Epidemiologia (CENEPI). A prevalência nacional em 1998 foi de 4,86 por 10 mil habitantes (média), e a detecção 2,60 por 10 mil habitantes (muito alta). No Maranhão, a prevalência foi de 16,13 por 10 mil habitantes (muito alta) e a detecção 7,94 por 10 mil habitantes (hiperendêmica) (MS, 2000).

A taxa de detecção de casos novos de hanseníase em menores de 15 anos determina a tendência secular desta endemia (MS, 1994). Em 1998, o Brasil teve valor muito alto deste coeficiente $(0,78$ por 10 mil habitantes) e o Maranhão apresentou hiperendemicidade do mesmo (3,93 por 10 mil habitantes) (MS, 2000).

Segundo o MS (1999), 88\% da população maranhense habita áreas de acesso a atividades do Programa de Controle de Hanseníase, e $66 \%$ dos municípios do estado são cobertos pelo mesmo. Avaliando o PEL, o MS (1998) propõe intervenção seletiva em áreas hiperendêmicas, pois dos 3.548 municípios com Programa de Hanseníase, 800 representam $80 \%$ da doença no Brasil.

Barreto et al. (1996) atestaram tendência de aumento da hanseníase, com destaque para o Nordeste do Brasil, refletindo tanto a melhoria na capacidade de detecção de casos novos quanto a expansão desta doença no País. Para Sabroza et al. (1993), o aumento das taxas de detecção da hanseníase no Brasil decorre da incapacidade de as ações de saúde realizadas limitarem a expansão da sua transmissão.

Este trabalho tem por objetivo descrever e analisar aspectos da detecção de casos novos de hanseníase em São Luís de 1993 a 1998, explicitando especificidades locais desta endemia. Foi analisada a tendência de morbidade da hanseníase por meio dos coeficientes de detecção geral e específicos por sexo, forma clínica e faixa etária.

\section{Material e métodos}

A partir de 1993 foi informatizado o registro das notificações de casos novos de hanseníase no Maranhão. O banco de dados das notificações de São Luís, desde então até 1998 (ano mais recente com dados disponíveis), foi obtido junto à Fundação Nacional de Saúde (FUNASA), contendo 3.930 registros. Foi definida como objeto de estudo deste trabalho a população de pacientes residentes em São Luís e diagnosticados pela primeira vez com hanseníase no período de 1993 a 1998. Foram excluídos 1.134 registros não correspondentes a este perfil. Destes, 925 por serem de residentes de outros municípios diagnosticados em São Luís e 66 pela falta de dados referentes ao local de moradia. Os demais 143 foram retirados por terem sido notificados em duplicidade ou como transferência, recidiva, detecção anterior a 1993 ou não referente a caso novo. Foi criado banco de dados dentro do programa Epi Info (versão $6.04 \mathrm{~b}$ ) para as 2.796 notificações restantes.

Os dados demográficos relativos à distribuição da população por sexo e faixa etária no período estudado foram obtidos na Fundação Instituto Brasileiro de Geografia e Estatística (IBGE, 2000).

Em relação aos grupos etários, respeitou-se a divisão adotada pelo MS, qual seja, de zero a 14 anos e igual ou maior de 15 anos. A faixa etária acima de 15 anos foi subdividida em grupos menores (15 a 19 anos, 20 a 39, 40 a 59 e a partir de 60 anos) para permitir análise mais aprofundada dos grupos populacionais mais acometidos pela doença.

As formas clínicas de hanseníase foram relatadas de acordo com a classificação de Madri, adotada no Brasil pelo MS (indeterminada, tuberculóide, virchowiana e dimorfa) (MS, 1994).

Para avaliação dos coeficientes de detecção foram usados parâmetros preconizados pelo CENEPI. Assim, foi considerado hiperendêmico o coeficiente superior a 4,0 por $10 \mathrm{mil} \mathrm{habi-}$ tantes; muito alto de 2,0 a 3,9 por 10 mil habitantes; alto de 1,0 a 1,9 por 10 mil habitantes; médio de 0,2 a 0,9 por 10 mil habitantes; e baixo inferior a 0,2 por 10 mil habitantes. A detecção em menores de 15 anos foi chamada hiperendêmica quando acima de 1,0 por $10 \mathrm{mil} \mathrm{ha-}$ bitantes; muito alta de 0,5 a 0,99 por 10 mil habitantes; alta de 0,25 a 0,49 por 10 mil habitantes; média de 0,05 a 0,24 por 10 mil habitantes; e baixa quando inferior a 0,05 por $10 \mathrm{mil} \mathrm{habi-}$ tantes (MS, 2000).

Os coeficientes de detecção foram calculados com seus intervalos de confiança (IC) de 95\% no programa Stata. O nível de significân- 
cia crítico assumido para rejeição da hipótese de nulidade adotado em todo o tratamento estatístico foi de uma probabilidade máxima de erro tipo I de $5 \%(P<0,05)$. Assumiu-se a distribuição binomial quando o número de casos relatados foi igual ou superior a 100, e a distribuição de Poisson quando houve número inferior de casos novos.

Como indicador de tendência de endemia proposto pela Divisão Nacional de Dermatologia Sanitária (DNDS), foi calculada a proporção de casos da forma tuberculóide em relação à soma dos casos tuberculóides, dimorfos e virchowianos (MS, 1986).

Para análise da tendência temporal da hanseníase em São Luís, os coeficientes de detecção gerais e desdobrados por sexo, forma clínica e faixa etária foram submetidos a vários modelos de regressão. Na análise específica dos coeficientes por forma clínica, sexo e idade não foram incluídos os dados de 1998, em que houve decréscimo da detecção local, estadual e nacional por problemas no sistema de informação (Brasil, 2000). Procurou-se assim evitar um provável viés de aferição. Os dados foram avaliados no programa Bio-Estat 2.0 pelos modelos de ajuste linear, exponencial, geométrico e logarítmico, sendo selecionado aquele de coeficiente de determinação mais alto para cada situação (Ayres et al., 2000).

\section{Resultados}

A Tabela 1 mostra a detecção da hanseníase hiperendêmica em São Luís e no Maranhão no período estudado, no qual a detecção nacional manteve-se muito alta. A detecção em São Luís cresceu de modo significante em 1997 e decresceu em 1998. Em 1998, houve decréscimo no coeficiente nacional de detecção de hanseníase, que vinha crescendo anualmente desde 1995. Somente em 1998 pode-se afirmar ter havido diferença estatisticamente significante entre os dados locais e estaduais, com taxas menores em São Luís. O contraste entre estes dados e os nacionais permaneceu estável.

De acordo com a Tabela 2, todas as taxas observadas foram hiperendêmicas, exceto as observadas para o sexo feminino nos dois primeiros anos, que podem ter sido muito altas. $\mathrm{O}$ decréscimo das detecções por sexo observado em São Luís em 1994, pode ser atribuído ao acaso, em contraste com o declínio significante destas taxas em 1998. Pode-se afirmar que de 1993 a 1996 a hanseníase foi mais freqüente nos homens do que nas mulheres de São Luís. A partir de 1997 não é possível assegurar ter havido diferença na detecção por sexo. Em relação ao ano anterior, pode-se afirmar que em 1997 houve crescimento significante da taxa de detecção da hanseníase em mulheres. O mesmo não pode ser dito em relação à detecção em homens. Dessa forma, o aumento da detecção geral em 1997 deveu-se ao seu componente feminino.

Optou-se por avaliar a tendência destes coeficientes de detecção de 1993 a 1997, por meio do modelo de regressão mais adequado a cada um deles. Houve estimativa de crescimento anual da detecção geral da hanseníase em São Luís de 0,1299 casos por 100 mil habitantes, atribuível também ao seu componente feminino segundo o modelo de ajuste exponencial (ambas com $P=0,0261)$. A detecção nacional teve estimativa de crescimento anual de 0,0662 casos por 100 mil habitantes segundo este modelo de ajuste ( $\mathrm{P}=0,0126)$, mas o mesmo não pode ser afirmado em relação à detecção no Maranhão (estimativa de crescimento anual de 0,6940 casos por 100 mil habitantes;

Número de casos novos de hanseníase, população residente e coeficientes de detecção (por 10 mil habitantes) em São Luís, no Maranhão e no Brasil, de 1993 a 1998.

\begin{tabular}{|c|c|c|c|c|c|c|c|c|c|}
\hline \multirow[t]{2}{*}{ Ano } & \multicolumn{3}{|c|}{ São Luís } & \multicolumn{3}{|c|}{ Maranhão } & \multicolumn{3}{|c|}{ Brasil } \\
\hline & Casos & População & Detecção (IC 95\%) & Casos & População & Detecção (IC 95\%) & Casos & População & Detecção (IC 95\%) \\
\hline 1993 & 392 & 738.326 & $5,31(4,80-5,84)$ & 3.002 & 5.088 .909 & $5,90(5,70-6,11)$ & 32.887 & 151.556 .521 & $2,17(2,15-2,20)$ \\
\hline 1994 & 370 & 748.786 & $4,94(4,44-5,48)$ & 2.632 & 5.160 .987 & $5,10(4,89-5,28)$ & 33.051 & 153.726 .463 & $2,15(2,13-2,18)$ \\
\hline 1995 & 424 & 758.983 & $5,59(5,05-6,15)$ & 3.023 & 5.231 .256 & $5,78(5,58-5,98)$ & 35.922 & 155.822 .296 & $2,30(2,28-2,33)$ \\
\hline 1996 & 500 & 780.833 & $6,40(5,83-6,99)$ & 3.490 & 5.222 .183 & $6,68(6,46-6,92)$ & 39.928 & 157.070 .163 & $2,54(2,51-2,57)$ \\
\hline 1997 & 630 & 801.896 & $7,86(7,26-8,51)$ & 4.545 & 5.295 .452 & $8,58(8,36-8,81)$ & 44.435 & 159.636 .413 & $2,78(2,75-2,81)$ \\
\hline 1998 & 480 & 819.636 & $5,86(5,34-6,40)$ & 4.254 & 5.356 .853 & $7,94(7,73-8,16)$ & 42.055 & 161.790 .311 & $2,60(2,57-2,63)$ \\
\hline
\end{tabular}


Tabela 2

Número de casos novos de hanseníase, população residente e coeficientes de detecção (por 10 mil habitantes) segundo sexo. São Luís, Maranhão, Brasil, de 1993 a 1998.

\begin{tabular}{lllllll}
\hline Ano & Casos & $\begin{array}{c}\text { Homens } \\
\text { População }\end{array}$ & Detecção (IC 95\%) & Casos & $\begin{array}{c}\text { Mulheres } \\
\text { População }\end{array}$ & Detecção (IC 95\%) \\
\hline 1993 & 226 & 343.863 & $6,57(5,74-7,50)$ & 166 & 394.463 & $4,21(3,60-4,91)$ \\
1994 & 207 & 348.735 & $5,94(5,15-6,78)$ & 163 & 400.051 & $4,07(3,47-4,76)$ \\
1995 & 231 & 353.484 & $6,53(5,71-7,41)$ & 193 & 405.499 & $4,76(4,10-5,48)$ \\
1996 & 280 & 362.439 & $7,73(6,84-8,69)$ & 220 & 418.394 & $5,26(4,59-6,00)$ \\
1997 & 326 & 371.441 & $8,78(7,83-9,80)$ & 304 & 428.786 & $7,09(6,32-7,92)$ \\
1998 & 251 & 380.450 & $6,60(5,80-7,48)$ & 229 & 439.186 & $5,21(4,56-5,95)$ \\
\hline
\end{tabular}

$\mathrm{P}=0,0888)$ e à observada nos homens de São Luís (estimativa de crescimento anual de 0,6210 casos por 100 mil habitantes, $\mathrm{P}=0,0591$ ) segundo o modelo de ajuste linear. Com relação a estas, é mais apropriado falar em tendência de estabilização, pois não apresentaram evidência de decréscimo.

A Tabela 3 não mostrou diferença estatística entre as detecções gerais por forma clínica em 1993. Em 1994, a detecção da forma virchowiana superou a observada nas formas indeterminada e dimorfa, mas não a da hanseníase tuberculóide. Em 1995, o coeficiente de detecção da forma tuberculóide ultrapassou os demais, menos o da forma virchowiana. A partir de 1996, a detecção da forma tuberculóide foi maior que as detecções das demais formas da hanseníase. Nesse ano não se pode afirmar ter havido diferença entre as detecções das formas virchowiana e indeterminada da hanseníase, tendo sido ambas maiores que a da hanseníase dimorfa. Em 1997 e 1998, desapareceram as diferenças entre as detecções destas três formas clínicas da hanseníase. Em nenhum momento as formas dimorfa e indeterminada foram as mais freqüentemente diagnosticadas.

A análise estatística dos coeficientes de detecção por forma clínica de 1993 a 1997, demonstrou crescimento na detecção da hanseníase indeterminada pelo modelo de ajuste linear (estimativa de crescimento anual de 0,1590 casos por 100 mil habitantes, $\mathrm{P}=0,0337)$. $\mathrm{O}$ modelo exponencial revelou estimativa de crescimento anual de 0,1981 casos por $100 \mathrm{mil} \mathrm{ha-}$ bitantes para a hanseníase tuberculóide ( $\mathrm{P}=$ 0,0062 ) e 0,0605 casos por 100 mil habitantes para a virchowiana $(P=0,0384)$. Não foi verificado crescimento na detecção da hanseníase dimorfa $(\mathrm{P}=0,8932)$.

Observando-se a Tabela 4, notou-se permanente hiperendemicidade da detecção abaixo de 15 anos. Suas oscilações anuais entre 1993 e 1998 poderiam ser atribuídas ao acaso. Só houve diferença clara entre os valores destas taxas com a superioridade de 1997 em relação a 1994. Seus percentuais em relação ao total diagnosticado foram, cronologicamente, os seguintes: $22,1 \%, 17,3 \%, 16,7 \%, 17,6 \%, 15,5 \%$ e $14,7 \%$.

A análise de tendência da detecção da hanseníase por faixa etária em São Luís de 1993 a 1997 pelo modelo de ajuste exponencial revelou crescimento significante nas faixas de 15 a 19 anos (estimativa de crescimento anual de 0,0980 casos por 100 mil habitantes, $\mathrm{P}=0,0078$ ); 20 a 39 (estimativa de crescimento anual de 0,0969 casos por 100 mil habitantes, $\mathrm{P}=0,0493$ ) e 40 a 59 anos (estimativa de crescimento anual de 0,1330 casos por 100 mil habitantes, $\mathrm{P}=$ 0,0385). O modelo de ajuste linear não demonstrou o mesmo nas faixas de zero a 14 anos ( $\mathrm{P}=$ $0,2751)$ e a partir de $60(\mathrm{P}=0,5748)$.

A proporção de casos novos da forma tuberculóide em relação à soma de casos novos das formas tuberculóide, virchowiana e dimorfa teve os seguintes valores em ordem cronológica no período estudado: $0,33,0,36,0,4,0,48$, 0,47 e 0,5 . Houve crescimento constante desta proporção ao longo do tempo estudado, exceto por pequena queda em 1997. A estimativa de crescimento anual desta proporção pelo modelo de ajuste logarítmico foi de $3,44 \%$, com valor de $\mathrm{P}$ extremamente significante $(0,0021)$.

\section{Discussão}

Brasil (2000) atribui a queda da detecção nacional em 1998 a problemas no sistema de informação, considerando este achado não significante do ponto de vista epidemiológico. Em favor desta hipótese, lembre-se que naquele ano ainda estava ocorrendo a substitui- 
Tabela 3

População residente, número de casos novos e coeficientes de detecção de hanseníase segundo forma clínica (por 10 mil habitantes). São Luís, Maranhão, Brasil, de 1993 a 1998.

\begin{tabular}{|c|c|c|c|c|c|c|c|c|c|}
\hline \multirow[t]{3}{*}{ Ano } & \multirow{3}{*}{ População } & \multicolumn{8}{|c|}{ Forma clínica } \\
\hline & & \multicolumn{2}{|c|}{ Indeterminada } & \multicolumn{2}{|c|}{ Tuberculóide } & \multicolumn{2}{|c|}{ Virchowiana } & \multicolumn{2}{|c|}{ Dimorfa } \\
\hline & & Casos & Detecção (IC 95\%) & Casos & Detecção (IC 95\%) & Casos & Detecção (IC95\%) & Casos & Detecção (IC 95\%) \\
\hline 1993 & 738.326 & 73 & $0,99(0,77-1,24)$ & 105 & $1,42(1,16-1,72)$ & 105 & $1,42(1,16-1,72)$ & 109 & $1,48(1,22-1,78)$ \\
\hline 1994 & 748.786 & 68 & $0,91(0,71-1,15)$ & 109 & $1,46(1,20-1,76)$ & 119 & $1,59(1,32-1,90)$ & 74 & $0,99(0,77-1,24)$ \\
\hline 1995 & 758.983 & 96 & $1,26(1,02-1,54)$ & 134 & $1,77(1,48-2,09)$ & 120 & $1,58(1,31-1,89)$ & 74 & $0,98(0,76-1,22)$ \\
\hline 1996 & 780.833 & 119 & $1,52(1,26-1,82)$ & 183 & $2,34(2,02-2,72)$ & 126 & $1,61(1,35-1,93)$ & 72 & $0,92(0,72-1,16)$ \\
\hline 1997 & 801.896 & 119 & $1,48(1,23-1,77)$ & 242 & $3,02(2,66-3,42)$ & 153 & $1,91(1,62-2,23)$ & 116 & $1,45(1,20-1,74)$ \\
\hline 1998 & 819.636 & 92 & $1,12(0,91-1,38)$ & 193 & $2,36(2,03-2,71)$ & 95 & $1,16(0,94-1,41)$ & 100 & $1,22(0,99-1,49)$ \\
\hline
\end{tabular}

Tabela 4

Número de casos novos de hanseníase, população residente e coeficientes de detecção (por 10 mil habitantes) segundo faixa etária. São Luís, Maranhão, Brasil, de 1993 a 1998.

\begin{tabular}{|c|c|c|c|c|c|c|}
\hline \multirow[t]{2}{*}{ Idade (anos) } & \multicolumn{6}{|c|}{ Ano } \\
\hline & 1993 & 1994 & 1995 & 1996 & 1997 & 1998 \\
\hline \multicolumn{7}{|l|}{$0-14$} \\
\hline Casos & 85 & 62 & 70 & 85 & 97 & 70 \\
\hline População & 275.486 & 279.753 & 283.564 & 255.342 & 262.231 & 268.033 \\
\hline Detecção (IC 95\%) & $3,09(2,47-3,82)$ & $2,22(1,70-2,84)$ & $2,47(1,92-3,12)$ & $3,33(2,66-4,12)$ & $3,70(3,01-4,51)$ & $2,61(2,04-3,30)$ \\
\hline \multicolumn{7}{|l|}{$15-19$} \\
\hline Casos & 47 & 55 & 59 & 66 & 83 & 72 \\
\hline População & 91.687 & 92.986 & 94.253 & 101.240 & 103.971 & 106.271 \\
\hline Detecção (IC 95\%) & $5,13(3,77-6,82)$ & $5,92(4,46-7,70)$ & $6,26(4,77-8,08)$ & $6,52(5,04-8,29)$ & $7,98(6,36-9,90)$ & $6,78(5,30-8,53)$ \\
\hline \multicolumn{7}{|l|}{$20-39$} \\
\hline Casos & 137 & 134 & 160 & 170 & 233 & 169 \\
\hline População & 245.255 & 248.729 & 252.116 & 270.229 & 277.518 & 283.659 \\
\hline Detecção (IC 95\%) & $5,59(4,69-6,22)$ & $5,39(4,52-6,38)$ & $6,34(5,40-7,41)$ & $6,29(5,39-7,29)$ & $8,40(7,34-9,52)$ & $5,96(5,10-6,91)$ \\
\hline \multicolumn{7}{|l|}{$40-59$} \\
\hline Casos & 74 & 71 & 99 & 110 & 156 & 107 \\
\hline População & 90.457 & 91.739 & 92.988 & 109.055 & 111.996 & 114.474 \\
\hline Detecção (IC 95\%) & $8,18(6,42-10,27)$ & $7,74(6,04-9,76)$ & $10,65(8,65-12,96)$ & $10,09(8,30-12,12)$ & $13,93(11,85-16,26)$ & $9,35(7,65-11,31)$ \\
\hline \multicolumn{7}{|l|}{$\geq 60$} \\
\hline Casos & 42 & 36 & 30 & 52 & 54 & 58 \\
\hline População & 35.081 & 35.579 & 36.062 & 41.175 & 42.285 & 43.225 \\
\hline Detecção (IC 95\%) & $11,97(8,63-16,18)$ & $10,12(7,09-14,01)$ & $8,32(5,61-11,88)$ & $12,63(9,43-16,56)$ & $12,77(9,59-16,66)$ & $13,42(10,19-17,34)$ \\
\hline
\end{tabular}


ção das fichas de notificação usadas nos estados pela ficha individual de investigação do Sistema de Informações sobre Agravos de Notificação (SINAN), de âmbito nacional. No Maranhão, esta ficha foi adotada durante 1997. Outro fato que reforça esta interpretação é a reprodução do decréscimo nacional em nível local e estadual.

Diante destas evidências, verificou-se que incluir as taxas de detecção referentes a 1998 prejudicaria as análises de tendência desta endemia por introduzir um provável viés de aferição, tendendo a minimizar a sua dimensão. Por esta razão, estas foram feitas referindo-se ao período 1993-1997.

O coeficiente de detecção médio em São Luís no período estudado foi de 5,99/10 mil habitantes, e o maranhense de 6,66/10 mil habitantes. Ao final de 1998, o Brasil ocupava o segundo lugar mundial em número de casos de hanseníase, superado pela Índia (WHO, 1999). A taxa de detecção deste país naquele ano foi de 5,90/10 mil habitantes, inferior, portanto, às médias ora mencionadas.

Meima et al. (1997) investigaram, por meio de estudo bibliográfico, a tendência das taxas de detecção de casos novos de hanseníase em áreas endêmicas do Pacífico, Ásia, África e América Latina. Concluíram que a única das áreas estudadas onde houve tendência consistente de aumento da detecção nos últimos 20 anos foi a América Latina. As tendências de aumento da detecção em São Luís e no Brasil se coadunam com os resultados destes autores.

Lombardi \& Suárez (1997) atribuem número maior de casos masculinos descritos à maior movimentação e oportunidade de contato dos homens e a exame clínico menos cuidadoso em mulheres. O aumento da detecção em mulheres de São Luís nos últimos anos estudados fez desaparecerem as diferenças estatisticamente relevantes na detecção por sexo. Isto concorda com a afirmação daqueles autores de que em comunidades onde as diferenças culturais mencionadas são menores não se encontram variações por sexo no número total de doentes. Reforça também este ponto de vista a tendência observada de crescimento da detecção local da hanseníase às custas do sexo feminino.

Irgens \& Skjaerven (1985) apontam tendência secular global da hanseníase com declínio da detecção e aumento na idade de início, dos casos masculinos e multibacilares. Considerando-se esta afirmação, o aumento do coeficiente de detecção encontrado em São Luís, associado ao aumento dos casos de formas paucibacilares e casos do sexo feminino falam a favor da existência de expansão da endemia.
O crescimento da detecção em São Luís deveu-se provavelmente mais à expansão da endemia que ao fortalecimento das suas atividades de controle. Esta proposição torna-se mais convincente diante do crescimento da forma tuberculóide, indicativo do acometimento de população capaz de desenvolver imunidade celular mais intensa contra o Mycobacterium leprae e portadora de resistência natural a este bacilo.

Os resultados de São Luís quanto à proporção de casos novos tuberculóides em relação à soma dos casos tuberculóides, dimorfos e virchowianos apontaram consistentemente para sua expansão, sendo sua pequena queda de 1997 logo superada no ano seguinte, apesar do número menor de casos detectados.

Pereira Jr. \& Torrecilla (1997) recomendam busca ativa na população em geral de áreas de alta prevalência (a partir de cinco casos por 10 mil habitantes) na profilaxia da hanseníase. Os coeficientes de prevalência têm valores superiores aos da detecção por incluírem casos antigos e novos. A precariedade do fluxo de informações relativas ao tratamento e alta dos pacientes impossibilitou o cálculo da prevalência em São Luís no período estudado. Entretanto, as taxas locais de detecção apontam prevalência que requer realização dessa atividade. Estes autores também indicam busca ativa de hanseníase pelo exame de escolares quando mais de $8 \%$ do total de casos detectados forem de menores de 15 anos, taxa amplamente ultrapassada em São Luís de 1993 a 1998.

Selvasekar et al. (1999) enfatizam a necessidade de inquéritos populacionais cuidadosos nesta faixa etária, tanto para detecção de casos como para seu melhor seguimento em áreas endêmicas. Encontraram mais de $80 \%$ dos pacientes com lesão única, a maioria detectada por exame de escolares e cerca de $30 \%$ com história de contato domiciliar com hanseniano, principalmente pais e avós. Reforçam este ponto de vista as conclusões do inquérito escolar de Koticha (1976), em que mais de 99\% dos casos não tinham deformidade quando diagnosticados e $86 \%$ estavam em estágios iniciais da hanseníase.

Em resumo, o que se observou em São Luís foi detecção hiperendêmica e com tendência de crescimento às custas do sexo feminino $\mathrm{e}$ das formas paucibacilares, principalmente da tuberculóide. Esta detecção cresceu na população de 15 a 59 anos, com maior expansão na faixa de 15 a 19 anos. Ficou demonstrada a necessidade de busca ativa na população menor de 15 anos, tanto pela hiperendemicidade de sua detecção quanto pelo porcentual destes pacientes em relação ao total de casos novos. 


\section{Referências}

AYRES, M.; AYRES Jr., M.; AYRES, D. L. \& SANTOS, A. S., 2000. Bio Estat 2.0: Aplicações Estatísticas nas Áreas das Ciências Biológicas e Médicas. Belém: Editora Sociedade Civil Mamirauá/Brasília: Conselho Nacional de Desenvolvimento Científico e Tecnológico.

BARRETO, M. L.; CARMO, E. H.; SANTOS, C. A. S. \& FERREIRA, L. A. D., 1996. "Emergentes", "re-emergentes" e "permanecentes": Tendências recentes das doenças infecciosas e parasitárias no Brasil. Informe Epidemiológico do SUS, 3:7-17.

BRASIL, M. T. L. R. F., 2000. A diminuição dos índices de detecção e a prevalência oculta. In: Congresso Brasileiro de Dermatologia, Anais, pp. 75-76, Salvador: Sociedade Brasileira de Dermatologia.

IBGE (Fundação Instituto Brasileiro de Geografia e Estatística), 2000. Censo Demográfico e Estimativas. 4 Maio $2000<$ http://www.ibge.net>.

IRGENS, L. M. \& SKJAERVEN, R., 1985. Secular trends in age at onset, sex ratio, and type index in leprosy observed during declining incidence rates. American Journal of Epidemiology, 4:695-705.

KOTICHA, K. K., 1976. Case detection through school surveys in Greater Bombay. Leprosy in India, 4 (Sup. 4):819-824.

LOMBARDI, C. \& SUAREZ, R. E. G., 1997. Epidemiologia da hanseníase. In: Hanseníase (S. Talhari \& R. G. Neves, org.), pp. 127-136, Manaus: Gráfica Tropical.

MEIMA, A.; GUPTE, M. D.; van ORRTMARSSEN, G. J. \& HABBEMA, J. D., 1997. Trends in leprosy case detection rates. International Journal of Leprosy and Other Mycobacterial Diseases, 3:305-319.

MS (Ministério da Saúde), 1986. Relatório dos Encontros Macrorregionais de Hanseníase: Avaliação de Desempenho. Brasília: Divisão Nacional de Dermatologia Sanitária, MS.
MS (Ministério da Saúde), 1994. Guia de Controle da Hanseníase. Brasília: Coordenação Nacional de Dermatologia Sanitária, Centro Nacional de Epidemiologia, Fundação Nacional de Saúde, MS.

MS (Ministério da Saúde), 1998. Hanseníase no Brasil: Progressos e Dificuldades em Relação à Eliminação. Brasília: Departamento de Gestão de Políticas Estratégicas, Secretaria de Políticas de Saúde, MS.

MS (Ministério da Saúde), 1999. Relatório de Atividades da Área Técnica de Dermatologia Sanitária: Ano de 1999. Brasília: Departamento de Gestão de Políticas Estratégicas, Secretaria de Políticas de Saúde, MS.

MS (Ministério da Saúde), 2000. Dados Epidemiológicos de 1998. Brasília: Coordenação Nacional de Dermatologia Sanitária, Centro Nacional de Epidemiologia, Fundação Nacional de Saúde, MS.

PEREIRA Jr., A. C. \& TORRECILLA, M. A. C., 1997. Profilaxia. In: Hanseníase (S. Talhari \& R. G. Neves, org.), pp. 151-152, Manaus: Gráfica Tropical.

SABROZA, P. C.; TOLEDO, L. M. \& OSANAI, C. H., 1993. A organização do espaço e os processos endêmico-epidêmicos. In: Saúde, Ambiente e Desenvolvimento: Processos e Conseqüências sobre as Condições de Vida (M. C. Leal, P. C. Sabroza, R. H. Rodriguez \& P. M. Buss, org.), v. 2., pp. 57-77, São Paulo: Editora Hucitec/Rio de Janeiro: ABRASCO.

SELVASEKAR, A.; GEETHA, J.; NISHA, K.; MANIMOZHI, N.; JESUDASAN, K. \& RAO, P. S., 1999. Childhood leprosy in endemic area. Leprosy Review, 1:21-27.

WHO (World Health Organization), 1999. Global leprosy situation. Weekly Epidemiological Record, 3:1-2.

Recebido em 2 de maio de 2001

Versão final reapresentada em 19 de junho de 2002

Aprovado em 8 de novembro de 2002 